\title{
OA03.04. An assessment of the effectiveness of acupuncture for the Trauma Spectrum Response: results of a rapid evidence assessment of the literature (REAL)
}

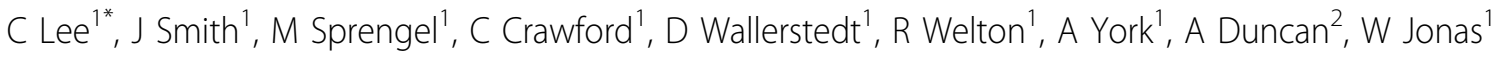 \\ From International Research Congress on Integrative Medicine and Health 2012 \\ Portland, Oregon, USA. 15-18 May 2012
}

\section{Purpose}

Exposure to deployment, combat, and other traumatic events can induce a spectrum of physical, cognitive, psychological and behavioral effects. This constellation has been termed the "Trauma Spectrum Response" (TSR) and includes depression, anxiety, post-traumatic stress disorder (PTSD), traumatic brain injury (TBI), sleep disturbances, substance abuse, cognition, headache, pain, and spirituality. Acupuncture has been shown to effectively treat such symptoms as headache, insomnia, stress, anxiety, depression, and perceived pain. While many literature reviews on acupuncture have been published, their conclusions are often difficult to interpret, inconsistent or contradictory. Thus, in order to comprehensively assess acupuncture's effectiveness across these symptoms, a rapid evidence assessment of the literature (REAL) was conducted to rigorously and systematically assess the quantity and quality of the existing systematic reviews/meta-analyses to gauge the effectiveness of acupuncture in treating the various components of TSR.

\section{Methods}

We conducted a search of systematic reviews published in the English language in several key databases (PubMed, Cochrane Database of Systematic Reviews, EMBASE, PsycINFO, and CINAHL) from inception to September 2011. Articles were included if they involved needle acupuncture as a treatment for at least one of the TSR components.

\section{Results}

We present the results of this review of systematic reviews for each of the TSR components and discuss the overall quality of these reports using the Scottish Intercollegiate Guidelines Network (SIGN) 50. Additionally, we present the results on the overall effectiveness of acupuncture and safety rating for each of the TSR components as determined by a group of subject matter experts using a systematic grading methodology.

\section{Conclusion}

The results of this review of reviews is expected to provide a systematic assessment of the quality of the current body of acupuncture literature for each of the TSR components and identify gap areas that may elicit further research.

\section{Author details}

${ }^{1}$ Samueli Institute, Alexandria, USA. ${ }^{2}$ Healingworks: Restoring and Renewing Military Families, Silver Spring, USA.

Published: 12 June 2012

doi:10.1186/1472-6882-12-S1-012

Cite this article as: Lee et al: OA03.04. An assessment of the effectiveness of acupuncture for the Trauma Spectrum Response: results of a rapid evidence assessment of the literature (REAL). BMC Complementary and Alternative Medicine 2012 12(Suppl 1):012.

${ }^{1}$ Samueli Institute, Alexandria, USA

Full list of author information is available at the end of the article

( 2012 Lee et al; licensee BioMed Central Ltd. This is an Open Access article distributed under the terms of the Creative Commons Attribution License (http://creativecommons.org/licenses/by/2.0), which permits unrestricted use, distribution, and reproduction in any medium, provided the original work is properly cited. 